\title{
Characteristics of flow velocities in the umbilical arteries, assessed by Doppler ultrasound
}

\author{
P.J.H.M. Reuwer ${ }^{1}$, W.C. Nuyen ${ }^{2}$, H.J.M. Beijer ${ }^{3}$, R.M. Heethaar ${ }^{4}$, H.W. \\ Bruinse $^{1}$, P. Stoutenbeek ${ }^{1}$ and A.A. Haspels ${ }^{1}$ \\ Departments of ${ }^{\prime}$ Obstetrics, ${ }^{2}$ Electronics, ${ }^{3}$ Exp. Peripheral Circulation and ${ }^{4}$ Medical Physics, University \\ Hospital, Utrecht, The Netherlands
}

Accepted for publication 8 March 1984

\begin{abstract}
REUWER, P.J.H.M., NUYEN, W.C., BEIJER, H.J.M., HEETHAAR, R.M., BRUINSE, H.W., STOUTENBEEK, P. and HASPELS, A.A. (1984): Characteristics of flow velocities in the umbilical arteries, assessed by Doppler ultrasound. Europ. J. Obstet. Gynec. reprod. Biol., 17, 397-408.

Measuring blood velocities by Doppler ultrasound has introduced a new noninvasive technique into obstetrical diagnostics. This study evaluates the information about fetoplacental hemodynamics that can be extracted from Doppler signals obtained from umbilical arteries.

The most significant blood flow characteristic is blood flow pulsatility expressed as pulsatility index (PI). The PI is the difference between systolic and diastolic velocity, divided by the mean velocity. The PI strongly correlates with downstream impedance to flow.

It is demonstrated that sophisticated spectral analysis of the Doppler signals has no practical advantages over the simple zero-crossing technique for establishing PI values, since blood flow in umbilical arteries has a blunt-to-flat profile.

This study shows that the PI is a gestational-age-related, intra- and inter-observer-reproducible variable. The significant decrease of $\mathrm{PI}$ values with advancing gestational age reflects a progressive reduction of placental vascular resistance to umbilical blood flow. The PI holds promise as a simple method for early diagnosis of impaired umbilical circulation.
\end{abstract}

pulsatility index; reproducibility; fetoplacental hemodynamics

\section{Introduction}

An adequate fetoplacental circulation is a prerequisite for normal fetal growth and well-being. The recent introduction of Doppler techniques for noninvasive assessment of blood velocities makes the human fetal circulation accessible to exploration. The combined use of imaging echo and pulsed Doppler ultrasound

Reprint requests: P.J.H.M. Reuwer, Department of Obstetrics and Gynecology, Univcrsity Hospital Utrecht, Catharijnsingel 101, 3511 GV Utrecht, The Netherlands. 
offers the theoretical possibility of blood volume flow measurements if the angle between the blood vessel and the Doppler ultrasound beam as well as the vessel diameter can be measured accurately. Most investigators focussed their attention on these quantitative flow measurements in the fetal aorta and venous duct. In practice, this technique is quite complex and laborious. In particular, errors in measuring the vessel cross-sectional area undermine the accuracy of quantitative flow measurements (Gill et al., 1981; Eik-Nes, 1980).

Doppler velocity signals, as such, are far easier to monitor, yielding pulsatile blood velocity patterns. Qualitative analysis of fetal blood velocity characteristics may reveal information about the functional state of the fetal circulation in terms of impedance to flow and cardiac performance (McDonald, 1974; McCallum et al., 1978; Stuart et al., 1980).

The present study evaluates the relevant information on fetoplacental hemodynamics that can be extracted from Doppler velocity recordings from umbilical arteries. The feasibility of different signal processing techniques was examined. The intra- and inter-observer reproducibility of the method was tested.

\section{Materials and methods}

\section{Location of the umbilical cord}

Women were examined in the semi-recumbent position. The umbilical cord was identified within the amniotic fluid using a linear array real-time scanner (Toshiba

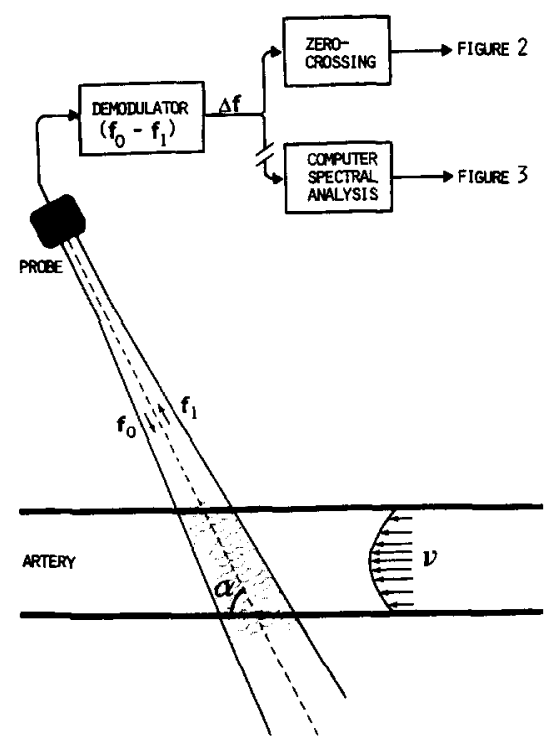

Fig. 1. Diagram of pulsed Doppler recording principle and signal processing techniques. The shaded area represents the Doppler sample window or volume. $f_{0}=$ emission frequency $(4 \mathrm{MHz}) ; f_{1}=$ reflected frequency; $V=$ blood velocity; $c=$ propagation velocity of sound in blood $(1530 \mathrm{~m} / \mathrm{s}) ; \Delta f=f_{0}-f_{1}=$ $2 V \cos \alpha \cdot f_{0} / c$. 
SAL-20A). After measuring the depth of the cord by electronic calipers, the real-time transducer was replaced by the probe of a pulsed Doppler device (ALVAR $4 \mathrm{MHz}$ ), held in the same direction.

\section{Doppler recording procedure}

The $10 \mathrm{kHz}$ repetition frequency with $1 \mu \mathrm{s}$ duration of emission resulted in a Doppler frequency reach up to $3.3 \mathrm{kHz}$. The range-gate facility of the pulsed Doppler instrument enables selection of a specific region (sample window or volume) from which Doppler shifts $(\Delta f)$ are received, without interference of echos from other vessels or the placenta along the ultrasound beam (Fig. 1). The sample window was fixed at 2,5 and $10 \mu \mathrm{s}$, respectively, corresponding to effective sample volume lengths of approximately $4.5,9.0$ and $16.5 \mathrm{~mm}$, and set at a depth to encompass the umbilical artery. Depending on the depth (between 2 and $6 \mathrm{~cm}$ ), the beamwidth $(-6 \mathrm{~dB})$ ranges from 2.1 to $4.7 \mathrm{~mm}$. When the sample volume is positioned within one of the 2 umbilical arteries, a pulsatile high-frequency Doppler signal is heard. When the sample volume partially covers the umbilical vein, an additional continuous low-frequency sound is easily recognized. By adjusting the direction of the probe and the depth of the sample volume, the best-quality arterial signals were selected by ear. Low-frequency Doppler shifts producted by probe movements or pulsations of the arterial walls were rejected by a $200 \mathrm{~Hz}$ filter. A recording was accepted for analysis if a steady state of at least 10 heart-beats had been obtained. Although fetal movements disturbed the steady state quite often, generally 3 or more acceptable recordings could be made within half an hour.

\section{Signal processing techniques}

The Doppler signal $(\Delta f)$ contains a spectrum of frequencies, corresponding to the unequal distribution of blood cell velocities (profile) across the vessel lumen. The average velocity across the vessel lumen (c.q. sample volume) can be assessed by 2

UMBILICAL ARTERY DOPPLER SHIFT
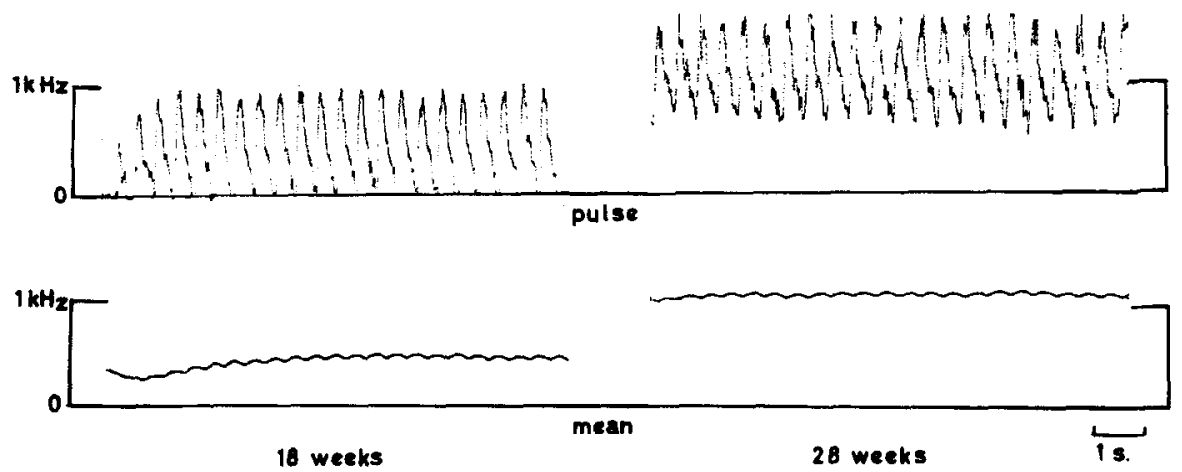

Fig. 2. Zero-crossing output. The upper tracing shows the pulsatile blood velocity (profile average) and the lower tracing shows the mean flow velocity (time-average). 
different signal-processing techniques (Fig. 1): 1, zero-crossing detection; 2, spectral analysis.

1. Zero-crossing detection: the built-in zero-crossing detector of the ALVAR instrument estimates the average of the multi-frequency signal and writes it as a function of time (Fig. 2). The pulsatile character of this average frequency, caused by the cardiac cycle, is clear. The non-pulsatile mean velocity (time-average) is established by low-pass filtering. Average systolic, diastolic and mean values are read from the paper strip.

2. Spectral analysis; the corresponding non-processed multi-frequency signals were recorded on an instrumentation recorder, for off-line analysis (Fig. 1).

The signal was analog-digital converted (eight-bit A/D converter with sample rate $6 \mathrm{kHz}$ ) into the memory of an Apple II computer. 16384 consecutive samples were stored $(2.73 \mathrm{~s})$. The stored sample values were divided into 64 blocks each of $0.043 \mathrm{~s}$, each containing 256 values. The power spectrum of each block was calculated with a Fast Fourier Transform (FFT) routine. The resulting 64 spectra each contained 128 spectral components and were smoothed by an eight-point running average.

An example of the result is shown in the upper panel of Fig. 3. The amount of
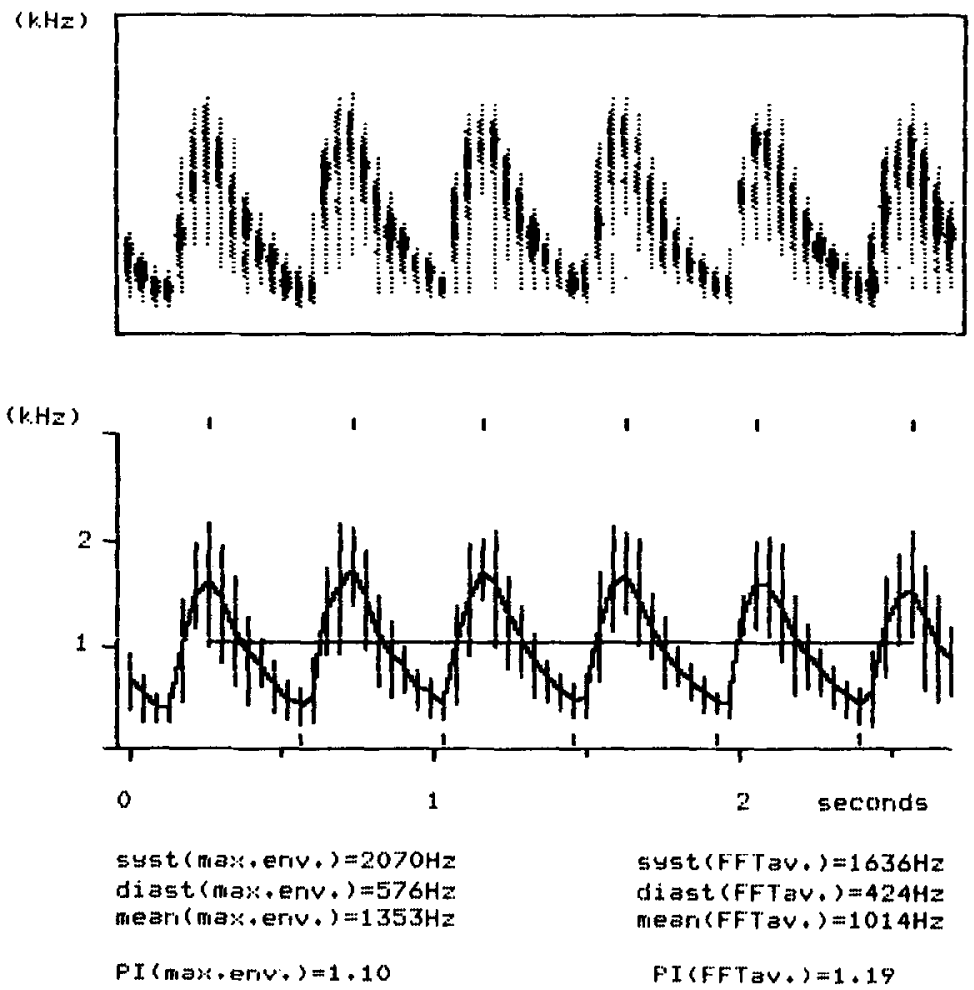

Fig. 3. Upper pannel: computer output after FFT analysis of the time-varying Doppler spectrum. Lower pannel: the same Doppler spectra after further data reduction. 
blood cells with a specific velocity is represented by the rate of blackening. The maximum envelope of the spectra represents the fastest-moving blood cells within the Doppler sample volume.

Next the average Doppler shift (center of gravity) of each spectrum was calculated (Fig. 3, lower panel). The spectral average of consecutive blocks represents the pulsatile average flow velocity as a function of time. Spectral width is indicated by vertical lines. Systolic and diastolic peaks were detected. Both for the spectral average and for the maximum envelope the following were calculated: the mean value of systolic peaks; the mean value of diastolic peaks; the mean velocity (time-average) over the maximum number of full cycles; the 'pulsatility index' (see next section).

The maximum envelope is assumedly a more reliable indicator (Gosling and King, 1974; Reneman, 1981) for the waveform of the blood velocity, since the maximum envelope represents the fastest-moving blood cells. It is therefore not disturbed by low-frequency changes of $\Delta f$ due to movements of the probe or vessel walls, nor by an erroneously measured umbilical venous component.

\section{Blood velocity characteristics}

Absolute values of Doppler frequency shifts $(\Delta f)$ depend on the angle between the ultrasound beam and the artery (Fig. 1). Because this angle is unknown, only angle-independent characteristics, such as ratios of Doppler shifts, yield information. Two angle-independent ratios were evaluated: 1, the pulsatility index (PI); 2, the profile ratio.

1. The pulsatility index; the PI is the difference between systolic and diastolic peaks, divided by the mean value over one cardiac cycle (Fig. 4). The PI quantifies pulse waveform damping and therefore reflects downstream impedance to flow (Gosling and King, 1974; Skidmore et al., 1980).

To test the accuracy of zero-crossing detection against complex spectral analysis, different PI values were calculated: the PI of the maximum envelope of the Doppler spectrum (PI max. env.), the PI calculated from the spectral average as assessed by spectrum analysis (PI FFTav) and as estimated by zero-crossing detection (PI $\varnothing$ ). It must be noted that the PIØ values were calculated over 10 or more heart-beats, whereas the FFT only covered $2.73 \mathrm{~s}$, corresponding to about 5 complete beats.

$$
\text { Pulsatility Index (PI) }
$$

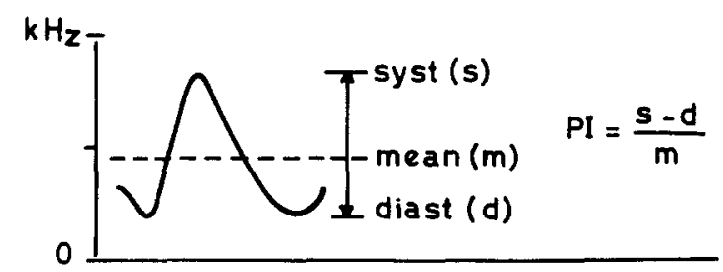

Fig. 4. Definition of PI after Gosling and King (1974). 
2. The profile ratio: blood velocity profiles within arteries may vary from flat to parabolic (McDonald, 1974). FFT spectral analysis reveals information on these profiles. A profile ratio can be calculated as the value of the maximum envelope divided by the spectral average value. The profile ratio equals 2 if flow has a parabolic laminar profile; i.e. the fastest blood cells have twice the average velocity (Fig. 7). If the profile ratio equals 1, blood flow has a flat front; i.e. turbulent or plug flow (McDonald, 1974).

Because arterial flow is pulsatile, the profile ratio might vary during the cardiac cycle. In order to examine this, the profile ratio calculated for the systole, i.e. (syst. max. env.)/(syst. FFTav), was compared with the mean (time-average) profile ratio, i.e. (mean max. env.)/(mean FFTav).

\section{Patients}

The Doppler recordings were obtained in 9 healthy women with normal singleton pregnancies. Three women were investigated at 18 , three at 28 and three at 38 wk of gestation. Gestational age was confirmed ultrasonically by first-trimester crown-rump length measurements. The women gave birth to healthy infants with weights appropriate for gestational age ( $\geqq P 10$ of the Amsterdam weight tables).

To test the reproducibility of the method, 18 or more measurements were made in each patient according to the protocol in Table $\mathrm{I}$.

\section{Statistics}

Possible differences in PI values, due to different signal processing techniques, were examined by calculating the correlation coefficients between PI(max. env), PI(FFTav.) and PIØ values, at each gestational age.

The influence of 4 variables on the reproducibility of the various PI values was evaluated: 1, gestational age; 2, observer; 3, moment of recording; 4, size of Doppler sample window. The (rank number) Krustal Wallis test was used for statistical analysis.

Doppler recordings using $2-\mu$ s sample windows, possibly sampling from only a part of the vessel cross-section, might yield profile ratios different from those from recordings using larger sample windows. Statistical analysis was performed using the Kruskal Wallis test.

TABLE I

Protocol for 18 repetitions in each patient

The sequence of observers A and B was randomized.

\begin{tabular}{llll}
\hline & Morning & Afternoon & Next morning \\
\hline Window $2 \mu \mathrm{s}$ & A-B & A-B & A-B \\
Window $5 \mu \mathrm{s}$ & A-B & A-B & A-B \\
Window $10 \mu \mathrm{s}$ & A-B & A-B & A-B \\
\hline
\end{tabular}




\section{Results}

At 18 wk of gestation blood velocity in the umbilical arteries consistently returned to zero during diastole, indicating a diastolic stop of flow. At 28 and 38 wk there was consistently a continuous forward flow throughout the cardiac cycle (Fig. 2). The PI values decreased significantly with advancing gestational age (Table II, Fig. 6).

The PI(FFTav.) values and the PI(max.env.) values as well as the PIØ values yielded significant linear correlations with slopes of about 1.0 (Fig. 5). Since the PIØ values were calculated over 10 or more heart-beats and the spectral analysis only covered about $S$ beats, small deviations may occur. During off-line analysis we were unable to sample exactly the same 5 heart-beats within a run of 10 or more cardiac cycles.

Repeated measurements in each patient resulted in a considerable variation in PI values at $18 \mathrm{wk}$, in contrast to smaller variations in patients of 28 and $38 \mathrm{wk}$ of gestation (Fig. 6). The variations ranged similarly for PIØ, PI(max.env.) and PI

\section{TABLE II}

Dependency of PIØ values on 4 possible sources for variation $P>0.05$ means no significance.

PI(max. env.) and PI(FFTav.) yielded similar $P$ values.

\begin{tabular}{llll}
\hline Gestational age & $18 \mathrm{wk}$ & $28 \mathrm{wk}$ & $38 \mathrm{wk}$ \\
\hline $18 \mathrm{wk}$ & - & $P<0.01$ & $P<0.01$ \\
$28 \mathrm{wk}$ & $P<0.01$ & - & $P<0.01$ \\
$38 \mathrm{wk}$ & $P<0.01$ & $P<0.01$ & - \\
Observers & $P=0.31$ & $P=0.65$ & $P=0.59$ \\
Moments & $P=0.04$ & $P=0.17$ & $P=0.24$ \\
Sample windows & $P=0.34$ & $P=0.43$ & $P=0.36$ \\
\hline
\end{tabular}
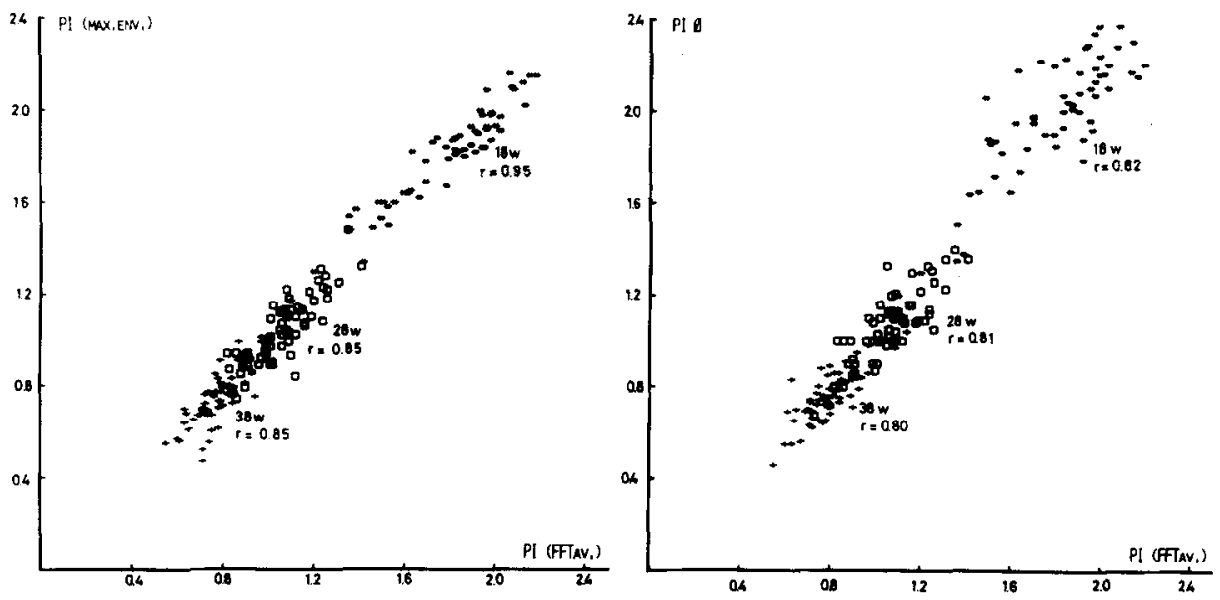

Fig. 5. Correlations between PI values obtained by different signal processing techniques. 
(FFTav.). No significant differences in PI values were found, between the 2 observers, or between recordings using different Doppler windows, or between the different moments of recording, except for a difference between recording moments at $18 \mathrm{wk}$ of gestation (Table II). The Kruskal Wallis test yielded a significantly different PI value in the afternoon as compared to PI values in the morning before and thereafter, suggesting a circadian rhythm. However, this difference (median PI
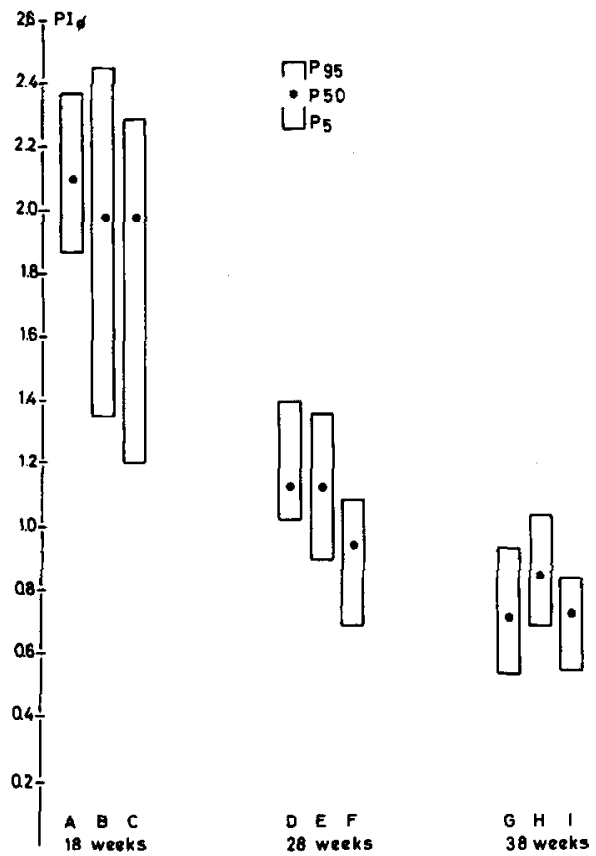

Fig. 6. Variation of PIØ values ( $n \geqq 18$ ) in each of the 9 patients (A-I).

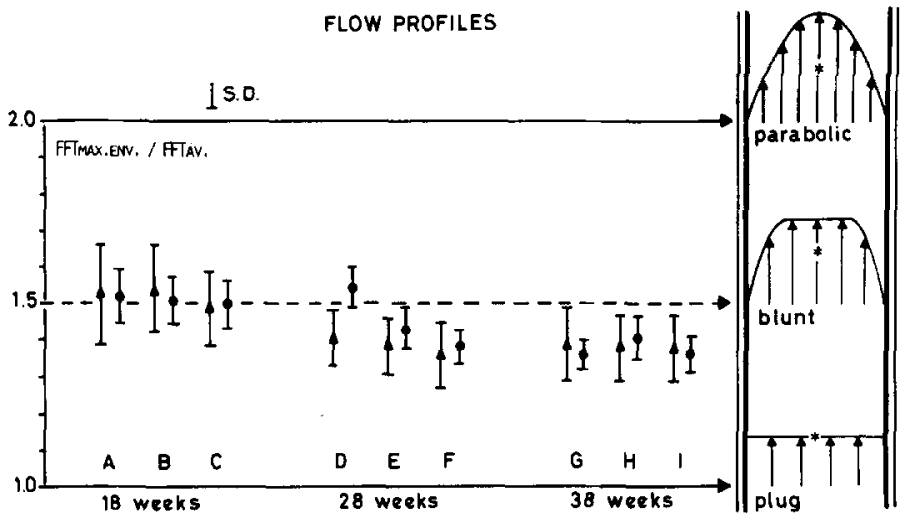

Fig. 7. The profile ratios in the umbilical artery in each of the 9 patients (A-I). $\Delta$, systolic profile ( $n \geqq 18) ; \ominus$, mean profile during the whole cardiac cycle $(n>=18)$. The diagram shows the flow profiles corresponding to profile ratios of $2,1.5$ and 1 , respectively. The asterisks in the diagram represent the average velocity of the profile. 
1.85 in the afternoon against 2.07 and 2.00 in the two mornings) was so small that we do not consider this difference of clinical significance.

The profile ratios of each of the nine patients are shown in Fig. 7, and indicate that blood flow in umbilical arteries has a blunt-to-flat profile. The profile ratios from the recordings with $2-\mu \mathrm{s}$ sample window were similar $(P=0.35)$ to those with larger windows. The systolic profile ratios did not differ from the mean profile ratios (i.e., the time-averaged velocity distribution during the whole cardiac cycle). The profile ratios at $18 \mathrm{wk}$ were higher than at 28 and $38 \mathrm{wk}$, but the proportional difference seemed too small to claim clinical relevance.

\section{Discussion}

Doppler signals from umbilical arteries contain information on the dynamics of fetal blood flow, both proximal (fetal cardiac) and distal (umbilical placental) to the site of recording. Actually, 3 angle-independent characteristics of the signals can be evaluated, each representing other aspects of the fetoplacental circulation: 1, pulsatility; 2 , blood flow profiles; 3 , velocity rate changes.

\section{Pulsatility}

Several studies on peripheral arteries in adults have shown that arterial blood flow pulsatility, expressed as PI, strongly correlates with downstream impedance to flow (Reneman, 1981). For example, the internal carotid artery, supplying a low-resistance vascular bed, has a PI of 2 (Gosling and King, 1974). In contrast, the PI of arteries in the legs while resting ranges from 5 to 15 and drops below 3 in the reactive hyperaemic phase after vessel occlusion (Skidmore et al., 1980) or after leg exercise (Gosling and King, 1974), in both cases due to arteriolar vasodilation.

The significant decrease of umbilical blood flow pulsatility (PI) with advancing gestational age in the present study is mainly determined by a rising diastolic velocity (Fig. 2). The emergence of a diastolic velocity level (McDonald, 1974) actually indicates a progressive reduction in placental vascular resistance, presumably due to ripening of the placental villi. Decreasing pulsatility with advancing pregnancy was previously demonstrated by Stuart et al. (1980). Our pilot studies (Reuwer et al., 1984) confirmed preliminary observations by McCallum (1978) of increased PI values during complicated pregnancies compared with normal pregnancies. These pilot studies strongly suggest that the PI holds promise as a method for early diagnosis of impaired fetoplacental circulation.

The present study demonstrates the intra- and inter-observer reproducibility of PI values. Repeated measurements in a patient do show some variation, particularly in early pregnancy (Fig. 6), but inter-observer differences, as well as recording on different moments of the day, as well as the use of different Doppler sample windows, could be excluded as sources for this variation. Some variation is to be expected, since repeated measurements are sampled at random from 1 of the 2 umbilical arteries each perfusing different parts of the placenta. The relatively pronounced PI variation at $18 \mathrm{wk}$ might be explained by greater asymmetry in placental development and unstable fetoplacental hemodynamics in immature gesta- 
tion relative to premature and term pregnancy. The remaining variation will be biological, resulting from varying vascular or uterine tone and hemodynamic alterations in the active fetus. To diminish the impact of incidental sources for variation, averaging PI values of several steady-state recordings must be recommended.

The simple zero-crossing technique gives satisfactory results as compared to the complex spectral analysis (Fig. 5). This is remarkable, since most investigators of larger peripheral arteries in adults prefer the Fast Fourier spectral analysis - in particular the maximum envelope - for reasons summarized by Gosling (1974) and Reneman (1981). First, Doppler shifts of venous origin, erroneously measured within the sample window, do not affect the spectral maximum envelope, whereas the spectral average (FFTav. or zero-crossing) will change. The present study, however, shows that the PI(max.env.) values equal the PI(FFTav.) values (Fig. 5). Apparently, the selection by ear of best-quality arterial signals is sufficiently accurate. Secondly, probe movements or vessel wall motions may induce lowfrequency artefacts in the zero-crossing output, and not in the maximum envelope of the FFT spectra. The present results show that these artefacts are easily avoided by the $200 \mathrm{~Hz}$ filter. Additional advantages of FFT spectral analysis, which are obvious in examinations of local obstructive arterial diseases (Gosling and King, 1974; Reneman, 1981), are not relevant with regard to obstetrical pathology. As far as PI values from umbilical arteries are concerned, no advantages of spectral analysis over zero-crossing detection can be demonstrated. The advantage of the zero-crossing technique is the reduction of the data to a simple tracing on paper, not limited in duration. This facilitates easy selection of steady state. Moreover, zero-crossing is inexpensive. Most commercially available Doppler devices are equipped with zerocrossing detectors. An additional on-line spectral analyser at least doubles the costs, whereas off-line spectral analysis by computer is complex and laborious.

\section{Blood flow profiles}

Doppler signals also contain information about arterial blood flow profiles, as explained before. This study shows that blood flow in umbilical arteries has a blunt-to-flat profile (Fig. 7). The proportional decrease of the profile ratios with advancing pregnancy is far less pronounced than the decrease of PI values. No evidence is found that the blunt profile changes during the cardiac cycle. This steady blunt profile explains why excellent correlations between PI(max.env.) and PI(FFTav.) values are found. The blunt profile also explains why the zero-crossing detector yields adequate PI values compared to spectral analysis, since the reliability of zero-crossing detectors increases with decreasing spectral width.

Blood flow profiles are mainly determined by the blood velocity and the diameter and curvatures of the artery (McDonald, 1974). Because the condition of the placental vascular bed has far more clinical relevance than the geometry of the umbilical arteries, the profile ratios are far less interesting for further clinical research than the PI.

\section{Velocity rate changes}

Theoretically, blood velocity signals from any artery also contain information about cardiac performance (McDonald, 1974). Stuart et al. (1980), studying Doppler 
sonograms of umbilical arteries, determined the acceleration slope of the systole, reflecting the force of fetal cardiac contraction. They found no significant changes in the course of pregnancy and concluded that there is a remarkably stable cardiovascular system during fetal growth in normal pregnancy. McCallum et al. (1978) determined the percentage of time during which blood velocity reaches its systolic peak, reflecting cardiac performance. They found no differences between normal and severely pathological pregnancies. These pilot studies do not encourage applications in future research.

In addition, a general remark regarding velocity rate changes should be made; while processing Doppler signals, a compromise must be made between the accuracy of average $\Delta f$ measurement and the velocity rate change that can be detected. The more accurate the $\Delta f$ detection for purposes of PI or profile ratio calculations, the more limited becomes the maximum velocity change that can be detected. The upper limit in most Doppler systems is about $20-25 \mathrm{~Hz}$. Faster velocity changes may be present but are not recognized. For these reasons we consider the practical value of Doppler velocity rate changes limited.

\section{General conclusions}

The recording procedure of Doppler signals from umbilical arteries is quite simple. Signal processing by the simple inexpensive zero-crossing technique gives satisfactory results as compared to the sophisticated and laborious signal processing by FFT spectral analysis, since blood flow in umbilical arteries has a steady, blunt-to-flat profile.

The most significant characteristic of blood velocity signals is pulsatility. The pulsatility index is an intra- and inter-observer-reproducible variable. The PI reflects placental resistance to umbilical blood flow. The PI decreases with advancing normal pregnancy and holds promise as a simple means for early diagnosis of Impaired fetoplacental circulation with subsequently poor fetal growth. An extensive study to establish reference PI values in normal pregnancy is in progress.

\section{Acknowledgements}

The authors wish to acknowledge the dedicated and skillful assistance of Marlene Zwinkels (Observer B) and to thank Hugo Kroeze for programming the computer spectral analysis.

\section{References}

Eik-Nes, S.H. (1980): Ultrasonic assessment of human fetal weight, growth and blood flow. Thesis, Malmö.

Gill, R.W., Trudinger, B.J., Garrett, W.J., Kossoff, G. and Warren, P.S. (1981): Fetal umbilical venous flow measured in utero by pulsed Doppler and B-mode ultasound. Amer. J. Obstet. Gynec., 139, $720-725$.

Gosling, R.G. and King, D.H. (1974): Continuous wave ultrasound as an alternative and complement to $X$-rays in vascular examinations, in: Cardiovascular Applications of Ultrasound, pp. 266-282. Editor:

R.S. Reneman. North-Holland/American Elsevier, New York. 
McCallum, W.D., Williams, C.S., Napel, S. and Daigle, R.E. (1978): Fetal blood velocity waveforms. Amer. J. Obstet. Gynec., 132, 425-429.

McDonald, A.D. (1974): Blood flow in arteries, 2nd Edn. Edward Arnold, London.

Reneman, R.S. (1981): What measurements are necessary for a comprehensive evaluation of peripheral circulation. Cardiovascular disease (Bulletin of the Texas Heart Institute) 8, 3, 435-454.

Reuwer, P.J.H.M., Stoutenbeek, P., Bruinse, H.W., Nuyen, W.C. and Heethaar, R.M. (1984): Doppler blood flow pulsatility in the umbilical arteries. Europ. J. Obstet. Gynec. reprod. Biol., 18, in the press. Skidmore, R., Woodcock, J.P., Wells, P.N.T., Bird, D. and Baird, R.N., (1980): Physiological interpretation of Doppler shift waveforms. III. Clinical results. Ultrasound Med. Biol., 6, 227-231.

Stuart, B., Drumm, J., FitzGerald, D.E. and Duignan, N.M. (1980): Fetal blood velocity waveforms in normal pregnancy. Brit. J. Obstet. Gynaec., 87, 780-785. 